\title{
Tissue Mimicking Phantoms for Microwave Brain Stroke Imaging
}

\author{
Sulayman Joof, Gokhan Cansiz, Selcuk Ozgur, Tuba Yilmaz, Mehmet Cayoren, and Ibrahim Akduman \\ Department of Electronics and Communication Engineering \\ Istanbul Technical University \\ Istanbul, Turkey \\ akduman@itu.edu.tr
}

\begin{abstract}
Within the past decade, once limited biomedical application of microwave imaging started to expand from the breast cancer imaging to imaging of other anomalies. One such anomaly is the brain stroke where the application of microwave imaging is two folds. One application is the identifying the source of stroke that is to categorize whether the stroke stems from blockage (ischemic) or bleeding (hemorrhagic). The other possible application is the continuous imaging of the progression of hemorrhagic stroke during the post-acute stage. In this work, a phantom for emulating the dielectric properties of the lossy brain tissue is given for testing of the microwave devices for continuous monitoring. The recipe is simple and is composed by mixing carboxymethyl cellulose, ethylene glycol, and deionized water. The recipe is simple, has viscose texture, and can be easily composed. Dielectric property measurements and comparison with the literature data is given in this paper.

Index Terms-microwave imaging, hemorrhagic stroke, dielectric properties, tissue mimicking phantoms
\end{abstract}

\section{INTRODUCTION}

Reports indicate that the new incidences of stroke in Europe is expected to increase by $34 \%$ by 2035 . In 2015 total cost off the disease was 45 billion euros and this cost is projected to increase. Currently the type of stroke is diagnosed with the conventional imaging systems such as Computed Tomography (CT) or Magnetic Resonance Imaging (MRI). During the postacute stage of the hemorrhagic stroke, the progression of the disease is monitored through CT or MRI. If the treatment is not optimized and timely, then the recovery and rehabilitation of the patient can be challenging. Therefore, to minimize both the recovery and rehabilitation time, accurate information regarding the progression of the disease is paramount.

Despite high resolution images, both CT and MRI can only provide discrete information about the progression of the disease. CT uses ionizing waves to image the progression of the disease. Thus, getting frequent CT scans is not advised for the patient. MRI scan requires long processing times. Both equipments are costly, and can be utilized for scanning different parts of the human body. Therefore, such equipments are subject to limited availability. Given the disadvantages neither CT scan nor MRI scan is suitable for continuous monitoring of hemorrhagic brain stroke.

This project has received funding from the European Union's Horizon 2020 research and innovation programme under the Marie Sklodowska-Curie grant agreement No 750346 .
Microwave imaging is an emerging scan technology that was mainly investigated for detection of breast cancer in the past decade. Research interest on new applications of this technology, specifically possibility of utilization of microwave imaging for both detection and continuous monitoring of hemorrhagic brain stroke is increasing [1], [2]. Microwave scanning equipment does not utilize ionizing waves, it is compact when compared to conventional bulky scanning units, due to low power outputs it does not require a shielded room; finally, microwave equipment is low cost comparing to CT and MRI. To this end, there is a need to exploit the potential utilization of microwave scanning devices for continuous monitoring of hemorrhagic brain stroke during post-acute stage. In this study we performed experiments with a gel-like phantom composed by mixing ethylene glycol (EG), carboxymethyl cellulose (CMC), and deionized water. The phantom is used for emulating the brain tissue for testing of the microwave brain monitoring prototype.

\section{METHODOLOGY}

\section{A. Dielectric Property Measurement}

Dielectric property measurements are performed with the open ended contact probe technique. The technique is generally utilized for measuring the dielectric properties of high permittivity and high conductivity materials, especially biological tissues. A slim form probe is suitable for measuring the dielectric properties of liquid or gel-like materials. However, the commercially available probes suffer from rapid wareoff. Therefore, an in house slim form probe is utilized for measuring the dielectric properties. The measurement set-up and the probe is shown in Fig 1a and Fig 1b, respectively. As shown in Fig 1b, the probe i fabricated with an RF cable and outer diameter of the coaxial probe is $2.2 \mathrm{~mm}$. The dielectric material between the inner and outer conductor is teflon and the impedance of the probe was $50 \mathrm{ohms}$.

Before the measurements are performed the probe is calibrated with the open, short, known liquid procedure. Deionized water is used as a known liquid. The dielectric properties are then measured by using the commercial Agilent's 85070 software. Please note that the dimensions of the in-house probe was compatible wit the commercial probe. Phantom material 


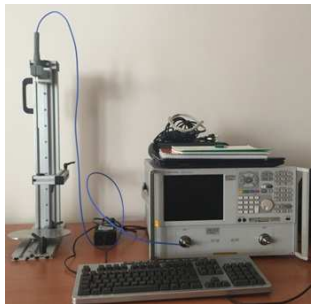

(a)

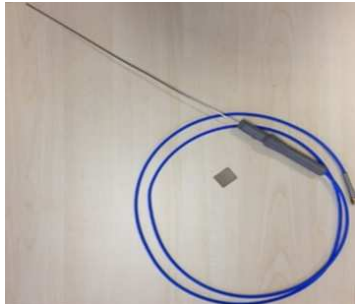

(b)
Fig. 1. Measurement Set-up and in house fabricated slim form probe (a) measurement set-up with Vector Network Analyzer and probe ; (b) slim form open ended contact probe designed and fabricated in house.

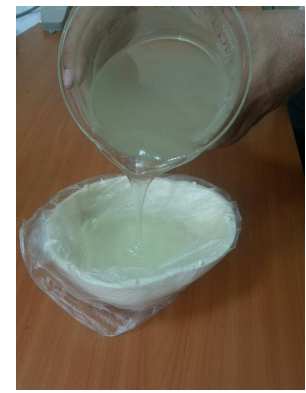

(a)

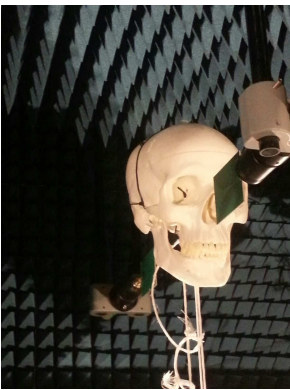

(b)
Fig. 2. Measurement set-up (a) phantom material; (b) microwave imaging set-up.

and microwave imaging set-up is shown in Fig $2 \mathrm{a}$ and Fig $2 \mathrm{~b}$, respectively.

\section{B. Recipe}

The phantom is composed by mixing $5 \% \mathrm{CMC}, 90 \% \mathrm{EG}$, and $10 \%$ deionized water. In this recipe CMC used as a gellifying agent. Deionized water is used as a solvent for the solidifying agent, that is CMC. Deionized water and Ethylene glycol are liquid; thus, mixed by volume. Solid CMC is then added by weight.

Due to the highly lossy nature of the brain and surrounding tissues, relatively lower frequencies with low power output is utilized for continuous monitoring of hemorrhagic brain stroke. To optimize the wave prorogation permittivity should match to the effective permittivity of human head and to prevent the wave attenuation the conductivity should be low a the frequency of interest. The matching medium should also possess long shelf life for practical applicability. In an earlier work by researchers same ingredients are used to characterized a matching medium [3].

\section{RESULTS}

Measurements are performed with different percentages of $\mathrm{CMC}$, EG, and deionized water. Permittivity and conductivity measurement results is given in Fig 3a and Fig 3b, respectively. The deionized water tends to emulate the dielectric properties of high water content biological tissues well. On the other hand, properties of EG does not directly emulate the dielectric properties of bodily tissues. From the measured

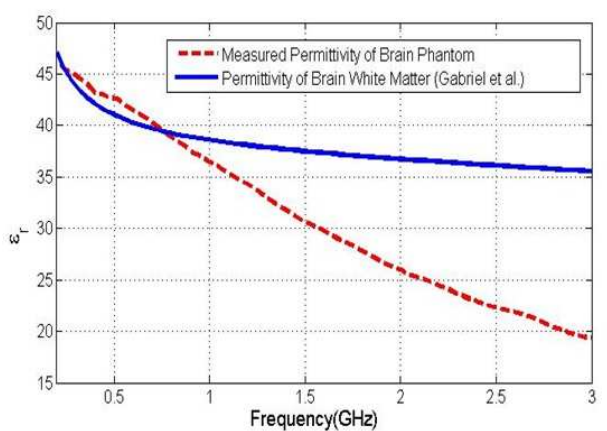

(a)

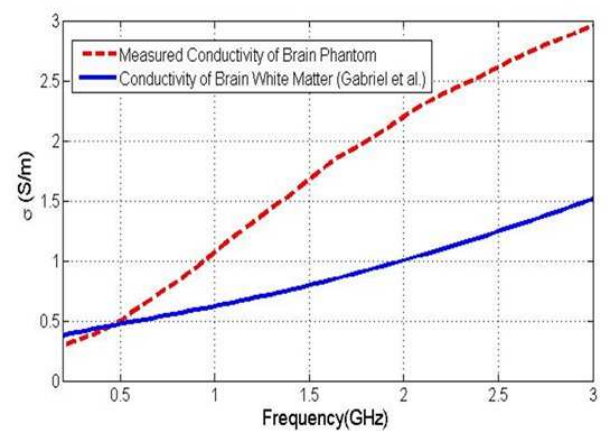

(b)

Fig. 3. Comparison of measured dielectric properties of the proposed phantom with the reference data [4], [5] (a) permittivity comparison ; (b)conductivity comparison.

dielectric properties it can be stated that the $\mathrm{CMC}$ increases the conductivity and Deionized water, having the highest dielectric properties in the frequency of interest, increases the dielectric property of the mixture.

\section{CONCLUSION}

A phantom emulating the dielectric properties of white matter is proposed. The phantom is composed with few ingredients and easy to compose. The shelf life of the phantom has not been investigated. Currently, other gellifying agents are investigated to decrease the conductivity of the phantom material.

\section{REFERENCES}

[1] Cayoren, Mehmet, and Ibrahim Akduman. "Continuous Monitoring of Hemorrhagic Strokes via Differential Microwave Imaging." Emerging Electromagnetic Technologies for Brain Diseases Diagnostics, Monitoring and Therapy. Springer, Cham, 2018. 37-57.

[2] Bilgin, E., et al. "Analysis of matching media effect on microwave brain stroke imaging via a spherically symmetrical head model." PIERS Proceedings, Prague, Czech Republic (2015).

[3] T. Yilmaz, H. Sahinturk, M. Acar and I. Akduman, "Matching medium characterization for microwave brain stroke imaging." 2016 IEEE International Symposium on Antennas and Propagation (APSURSI), pp.1485-1486, June 2016.

[4] Gabriel, Sami, R. W. Lau, and Camelia Gabriel. "The dielectric properties of biological tissues: II. Measurements in the frequency range 10 $\mathrm{Hz}$ to $20 \mathrm{GHz}$." Physics in medicine and biology 41.11 (1996): 2251.

[5] Gabriel, Sami, R. W. Lau, and Camelia Gabriel. "The dielectric properties of biological tissues: III. Parametric models for the dielectric spectrum of tissues." Physics in Medicine and Biology 41.11 (1996): 2271. 